\title{
Percepção de acompanhantes-usuários e enfermeiros sobre qualidade assistencial em Unidades Pediátricas*
}

\author{
Perception of companions-users and nurses about the quality of care in Pediatric Units \\ Percepción de acompañantes-usuarios y enfermeros sobre la calidad asistencial en Unidades \\ Pediátricas
}

Marta Maria Melleiro ${ }^{1}$, Daisy Maria Rizatto Tronchin ${ }^{2}$

\begin{abstract}
RESUMO
Objetivo: Descrever a percepção de acompanhantes-usuários e de enfermeiros de Unidades Pediátricas e de Terapia Intensiva Pediátrica Neonatal sobre a qualidade assistencial, pautada no modelo donabediano. Método: O local do estudo foi o Hospital Universitário da Universidade de São Paulo e a população constituiu-se de 22 enfermeiros e 192 acompanhantes. A coleta de dados ocorreu de agosto a outubro de 2006, por meio de um questionário. Os dados foram analisados, segundo os índices percentuais e testes estatísticos. Resultados: Os quesitos que apresentaram diferença estatística significante em estrutura, foram os referentes a recursos materiais, humanos e hotelaria; em processo, o relacionamento dos usuários com a equipe e a relevância dos registros de enfermagem, quanto ao resultado observou-se a importância das orientações para a alta e a satisfação com a assistência. Quanto à favorabilidade dos escores das três dimensões avaliadas verificou-se que a de estrutura obteve o menor escore. Conclusão: A dimensão de estrutura requer maior atenção dos gerentes desse serviço de saúde.

Descritores: Qualidade da assistência à saúde; Avaliação em saúde; Enfermeiras; Acompanhantes de pacientes
\end{abstract}

\begin{abstract}
Objective: This study aimed to describe the perception of companions-users and nurses about the quality of care; it was developed with nursing workers of Pediatric Units and Pediatric Neonatal Intensive Care. The study was based on the donabedian model .Method: The location of the study was the University Hospital of the University of Sao Paulo, and the population consisted of 22 nurses and 192 companions. Data collection occurred between August and October 2006 using a questionnaire. The data were analyzed using percentages and rates of the statistical tests. Results: The issues that were statistically significant in the area of structure were those related to materials and human resources and to hospital resources for accommodation; in the processing area was the relationship of users with the team and relevance of the nursing records; and, the result showed the importance of the guidelines for patient discharge and the satisfaction with care. In regard to the successful outcome of the scores of the three areas evaluated, it was found that the structure had the lowest score. Conclusion: The parameter structure requires greater attention from health service managers.
\end{abstract}

Descriptors: Quality of health care; Health services evaluation; Nurses; Patient escort service

\section{RESUMEN}

Objetivo: Este estudio tuvo como objetivo describir la percepción de acompañantes-usuarios y de enfermeros de Unidades Pediátricas y de Terapia Intensiva Pediátrica Neonatal sobre la calidad asistencial, pautada en el modelo donabediano. Método: El local del estudio fue el Hospital Universitario de la Universidad de Sao Paulo y la población estuvo constituida por 22 enfermeros y 192 acompañantes. La recolección de datos ocurrió entre agosto y octubre de 2006, por medio de un cuestionario. Los datos fueron analizados utilizando los índices de porcentajes y las pruebas estadísticas. Resultados: Los quesitos que presentaron diferencia estadística significante en el área de estructura fueron los que se referían a recursos materiales, humanos y hotelería; en el área de proceso fueron la relación de los usuarios con el equipo y la relevancia de los registros de enfermería; y, en lo que se refiere al resultado se observó la importancia de las orientaciones para el alta y la satisfacción con la asistencia. En lo que se refiere al resultado favorable de los puntajes de las tres dimensiones evaluadas se verificó que el de estructura obtuvo el menor puntaje. Conclusión: La dimensión de estructura requiere mayor atención de los gerentes de ese servicio de salud.

Descriptores: Calidad de la atención de salud; Evaluación de servicios de salud; Enfermeras; Acompañantes de pacientes

* Trabalho realizado no Hospital Universitário (HU) da Universidade de São Paulo USP - São Paulo (SP), Brasil.

1 Doutora em Enfermagem. Professora do Departamento de Orientação Profissional da Escola de Enfermagem da Universidade de São Paulo - USP - São Paulo (SP), Brasil.

2 Doutora em Enfermagem. Professora do Departamento de Orientação Profissional da Escola de Enfermagem da Universidade de São Paulo - USP - São Paulo (SP), Brasil. 


\section{INTRODUÇÃO}

A percepção dos usuários e dos profissionais de saúde, a respeito dos serviços oferecidos pelas instituições é considerada um importante aparato para o desenvolvimento dos processos assistenciais e gerenciais, à medida que possibilita aos gerentes desses serviços, revisarem suas metas e satisfazerem as expectativas dos usuários e de seus trabalhadores.

Nesse cenário, os dirigentes dos serviços de saúde passaram a instituir ações e programas de qualidade, capazes de propiciar a melhoria contínua da assistência prestada nesses estabelecimentos. Para tanto, a qualidade está, intrinsecamente, relacionada à satisfação, tanto dos trabalhadores como dos usuários.

No que tange aos usuários, conhecer seu grau de satisfação, suas impressões e opiniões relacionadas ao atendimento em saúde, constitui-se em uma ferramenta de gestão a ser utilizada no planejamento e aprimoramento da qualidade das ações de saúde ${ }^{(1)}$.

Com base na percepção do usuário, pode-se obter um conjunto de conceitos e atitudes relacionados ao atendimento recebido, que, por proporcionarem a reorganização das ações de saúde, representam uma das formas para se avaliar a qualidade dos serviços ${ }^{(2)}$.

$\mathrm{Na}$ saúde, a avaliação dos serviços envolve a coleta sistemática de informação sobre as atividades, características e resultados dos programas, recursos humanos e produtos à disposição de pessoas específicas, para redução das incertezas e tomar decisões em relação ao que esses programas realizam e qual seu impacto em uma determinada realidade ${ }^{(3-4)}$.

Nessa perspectiva, a avaliação é vista como função de gestão destinada a auxiliar o processo de tomada de decisão, visando a torná-lo mais racional. Assim, é preciso que se defina o objetivo da avaliação, tendo clareza para que esta seja feita, considerando-se como beneficiário final o usuário do serviço ou programa e não unicamente quem a solicitou ${ }^{(5)}$.

Com o propósito de atender às demandas dos usuários, os serviços de saúde devem ser avaliados, procurando-se responder às seguintes questões propostas por Donabedian ${ }^{(1)}$ : A infraestrutura existente atende às necessidades dos usuários? Os processos estão ocorrendo de maneira adequada? Os resultados obtidos são bons? Os usuários estão satisfeitos?

As respostas a essas indagações revelam que a qualidade não se constitui em um atributo abstrato, mas é construída pela avaliação de estrutura-processo-resultado ${ }^{(()}$.

$\mathrm{Na}$ avaliação dos serviços de saúde, outro aspecto diz respeito à bagagem conceitual dos trabalhadores atuantes nesses estabelecimentos, por deterem elementos intrínsecos ao processo avaliativo, como a percepção e a experiência profissional.
Dessa maneira, discutir qualidade como produto do trabalho das pessoas significa compreendê-las, bem como considerar suas percepções sobre os fenômenos organizacionais, buscando valorizá-las e incluí-las no processo para melhoria da qualidade dos serviços ${ }^{(7)}$.

Fundamentadas nessas premissas, delineou-se o objetivo deste estudo que foi o de descrever a percepção de acompanhantes-usuários e enfermeiros das Unidades de Pediatria e de Terapia Intensiva Pediátrica/Neonatal (UTIP/N), sobre a qualidade assistencial.

\section{MÉTODOS}

Trata-se de um estudo exploratório, descritivo de abordagem quantitativa. A investigação foi realizada nas Unidades de Pediatria e UTIP/N do Hospital Universitário da Universidade de São Paulo (HU-USP).

A população constituiu-se de 22 enfermeiros que atuavam nas referidas unidades e 192 acompanhantesusuários de crianças internadas.

O projeto foi aprovado pela Comissão de Ensino e Pesquisa e pelo Comitê de Ética em Pesquisa do HUUSP, respeitando-se os trâmites legais. O Termo de Consentimento Livre e Esclarecido (TCLE) foi elaborado, de acordo com a Resolução n. ${ }^{0} 196 / 96$.

A coleta de dados ocorreu de agosto a outubro de 2006, por meio de um questionário, pautado em revisão de literatura, com enfoque na qualidade e na avaliação de serviços, procurando-se, ainda, acrescentar assertivas que expressassem a vivência das pesquisadoras referente à temática descrita.

O instrumento constituiu-se de duas partes: a primeira, de dados sociodemográficos e a segunda, de proposições positivas e negativas, acerca da qualidade assistencial, empregando-se a Escala de Likert, contendo cinco graus, a saber: discordo totalmente (DT), discordo parcialmente (DP), indiferente (I), concordo parcialmente (CP) e concordo totalmente (CT).

Frente aos referenciais empregados para avaliar a qualidade em saúde, optou-se pelo modelo donabediano ${ }^{(1,6)}$, constituído pelos componentes de estrutura, processo e resultado.

A dimensão de estrutura compreendeu aspectos referentes aos recursos humanos (quantitativo de pessoal), materiais (insumos existentes) e hotelaria (mobiliário). Em processo, abordaram-se elementos relativos ao relacionamento do usuário com os profissionais de saúde, as orientações ministradas durante a internação e os registros de enfermagem no prontuário. Quanto ao resultado, evidenciou-se a satisfação do usuário e dos enfermeiros com a qualidade assistencial, a importância da participação do usuário no tratamento e a eficácia das orientações para a alta hospitalar.

O questionário foi considerado fidedigno, ao ser 
submetido à validação de conteúdo por sete especialistas e à consistência interna por meio do valor de Alpha de Cronbach $^{(8)}$.

As autoras fizeram um pré-teste do questionário, visando a assegurar sua compreensão, identificar suas possíveis falhas e realizar à sua reformulação. Salienta-se que não houve necessidade de alteração do instrumento, sendo esses entregues aos participantes do estudo com o TCLE.

Os dados foram armazenados em planilha eletrônica do programa Excel ${ }^{\circledR} 97$ e analisados por meio do sistema Statistic Package for Social Sciences $\left(S P S S^{\circledR}\right)$.

O tratamento dos dados foi realizado, segundo a estatística descritiva. Para comparar os escores nas três dimensões, foram empregados os testes de Friedman e Fisher.

\section{RESULTADOS}

Quanto à caracterização dos 192 acompanhantesusuários, observou-se que 174 (90,6\%) eram constituídos dos pais das crianças internadas. Em relação ao sexo, constatou-se que $160(83,3 \%)$ eram do sexo feminino e a média de idade foi de 29,6 anos.

No que tange aos enfermeiros, 21 (95,5\%) eram do sexo feminino, com idade média de 35 anos, 11,5 anos de experiência profissional e $14(63,7 \%)$ possuíam pósgraduação.

Relativo às dimensões avaliativas, estrutura, processo e resultado foram encontrados os achados a seguir. A dimensão de estrutura abarcou proposições sobre os recursos humanos, materiais e hotelaria hospitalar.

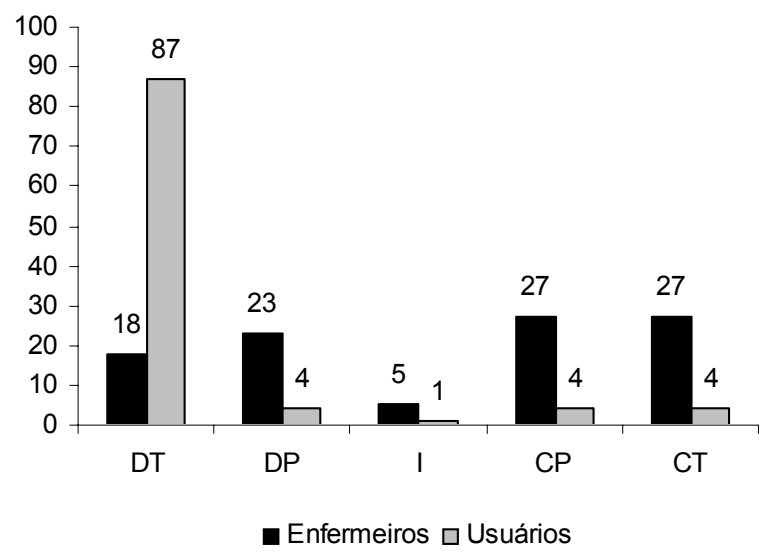

Teste Exato de Fisher $(\mathrm{p}<0,001)$

Figura 1 - Respostas relativas ao quantitativo de profissionais de enfermagem. HU-USP. São Paulo, 2006

$\mathrm{Na}$ Figura 1, considerando que para os acompanhantes-usuários, a assertiva proposta foi "Houve falta de pessoal de enfermagem para cuidar das crianças internadas", verificou-se que 167 (87\%) discordaram totalmente, indicando percepção positiva em relação ao quantitativo de pessoal. A resposta dos enfermeiros sobre essa assertiva, mostrou não haver tendência, visto que os percentuais de concordância e discordância mostraramse próximos.

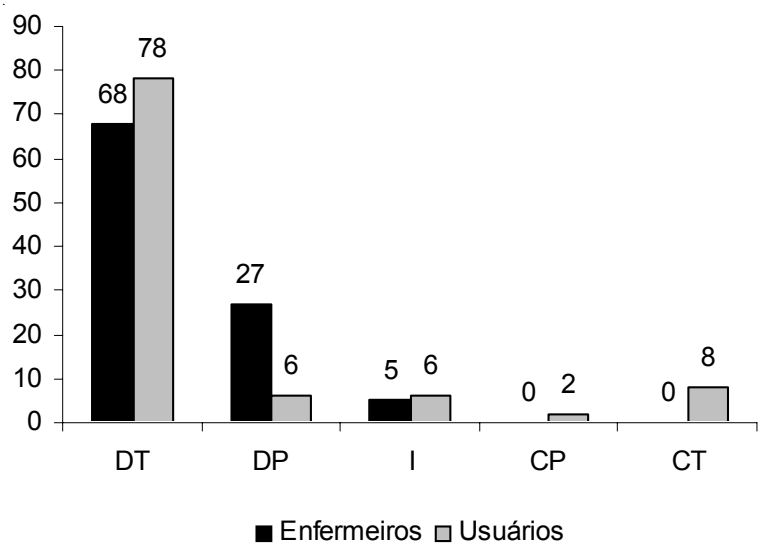

Teste Exato de Fisher $(\mathrm{p}<0,021)$

Figura 2-Respostas relativas aos recursos materiais. HUUSP. São Paulo, 2006

A análise do Figura 2 apontou para um percentual elevado de discordantes da assertiva, cuja conotação é negativa, "Há falta de material de consumo para o atendimento dos usuários". Assim, 150 (78\%) acompanhantes e 15 (68\%) enfermeiros discordaram totalmente.

Referente à hotelaria hospitalar, os participantes foram consultados, especificamente sobre o mobiliário existente na instituição. Averiguou-se que 136 (71\%) acompanhantesusuários consideraram-no adequado e que 9 (41\%) enfermeiros demonstraram restrições quanto a esse quesito, com significância estatística $\mathrm{p}<0,001$.

Em relação a processo, foram evidenciados aspectos concernentes ao relacionamento do usuário com os profissionais de saúde, às orientações ministradas durante a internação e aos registros de enfermagem no prontuário.

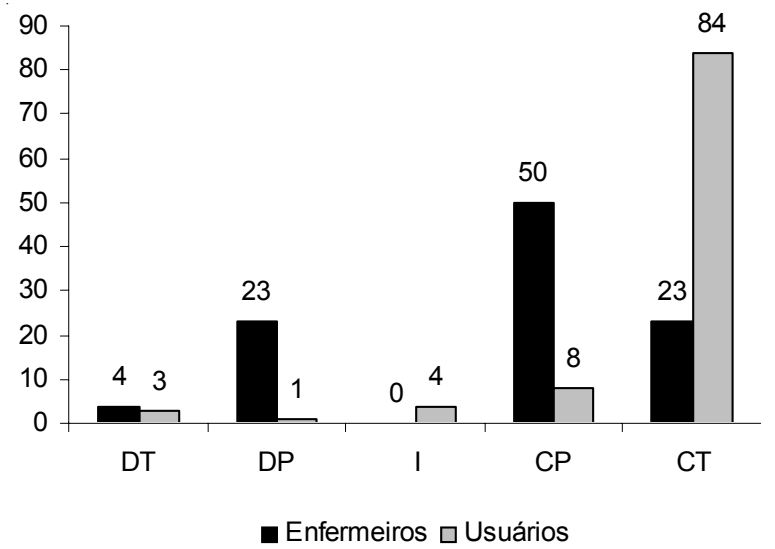

Teste Exato de Fisher $(p<0,001)$

Figura 3 - Respostas relativas ao relacionamento do usuário com os profissionais de enfermagem. HU-USP. São Paulo, 2006 
$\mathrm{Na}$ Figura 3, verifica-se que 168 (92\%) acompanhantesusuários (somatório de concordantes total e parcial) consideraram o relacionamento com a equipe de enfermagem um fator relevante na internação de suas crianças. O somatório do quantitativo de enfermeiros que concordou parcial e totalmente com essa assertiva perfez 16 (73\%).

Quanto às orientações para a saúde, evidenciou-se que $18(82 \%)$ enfermeiros e 128 (67\%) acompanhantesusuários demonstraram percepção positiva $(\mathrm{p}<0,892)$.

Para os registros da assistência prestada, os resultados mostraram que 15 (68\%) enfermeiros e 167 (87\%) acompanhantes-usuários apontaram que esses eram realizados de modo a qualificar o cuidado, sendo considerado estatisticamente significante $\mathrm{p}<0,01$.

No que tange à dimensão de resultado discutiu-se a satisfação do usuário e dos enfermeiros com a qualidade assistencial, a importância da participação do usuário no tratamento e a eficácia das orientações para a alta hospitalar.

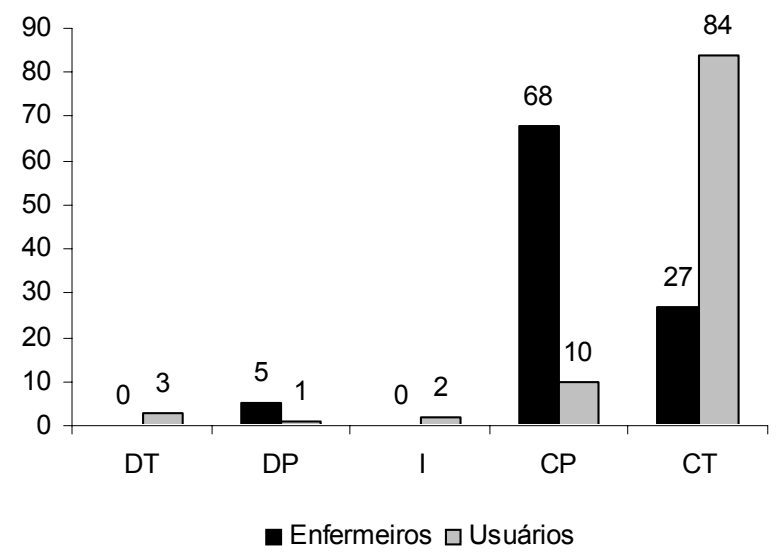

Teste Exato de Fisher $(\mathrm{p}<0,001)$

Figura 4 - Respostas relativas à satisfação com a qualidade assistencial HU-USP. São Paulo, 2006

Na Figura 4, observa-se que $21(95 \%)$ enfermeiros e $182(95 \%)$ acompanhantes-usuários mostraram-se parcial ou totalmente satisfeitos com a qualidade assistencial. Todavia, chama a atenção o fato de que 15 (68\%) enfermeiros terem concordado parcialmente.

Diante da participação do usuário no tratamento, identificou-se que 18 (82\%) enfermeiros e $109(57 \%)$ acompanhantes-usuários consideraram-na essencial $(\mathrm{p}<0,77)$.

Concernente às orientações para a alta, constatou-se que $21(96 \%)$ enfermeiros referiram que essas atendiam às necessidades dos usuários. Em contrapartida, averiguou-se que 96 (50\%) usuários demonstraram-se indiferentes, com diferença estatística significante $\mathrm{p}<0,001$.

A Figura 5 mostra que o escore médio dos enfermeiros em relação à dimensão de estrutura foi de 2,67 ( $\mathrm{dp} \pm$ $0,51)$, de processo $2,15(\mathrm{dp} \pm 0,60)$ e de resultado 3,45 $(\mathrm{dp} \pm 0,39)$. Referente aos acompanhantes-usuários, os valores encontrados foram de 1,63 (dp \pm 0,77), 3,76 (dp \pm
0,63) e 3,74 (dp $\pm 0,69)$, respectivamente. Dessa maneira, o escore de resultado foi melhor avaliado pelos enfermeiros, e o de processo pelos acompanhantesusuários.

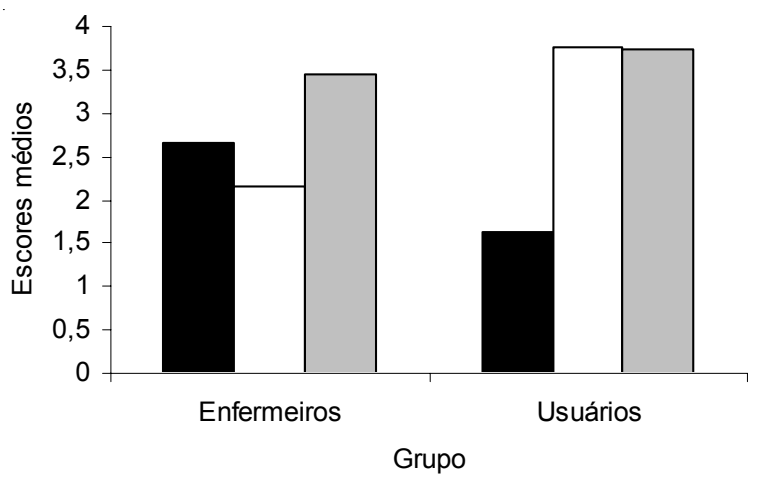

— Estrutura $\square$ Processo $\square$ Resultado

Teste não-paramétrico de Friedman $(\mathrm{p}<0,001)$.

Figura 5 - Valores de média e desvio-padrão, segundo escore de favorabilidade, HU-USP. São Paulo, 2006

Observou-se, ainda, que tanto no grupo de enfermeiros como no de acompanhantes-usuários houve diferença significante entre os três escores avaliados $(\mathrm{p}<$ $0,001)$.

\section{DISCUSSÃO}

Na caracterização dos acompanhantes-usuários, notouse a expressiva permanência dos pais durante a internação de seus filhos, corroborando com os autores ${ }^{(9-10)}$ ao referirem que os pais ao assumirem a condição de cuidadores garantem sua participação nos cuidados e sua operacionalização implica o desenvolvimento de ações pelos profissionais de saúde, agregando valor ao incluílos na perspectiva do cuidado.

Nesse sentido, com a permanência dos acompanhantes durante a internação das crianças, a equipe de enfermagem tem um papel de extrema importância, pois ambos acabam necessitando de sua atenção e, para tanto, esta deve incentivar a efetiva participação dos pais, de modo a torná-los aptos a prestarem os cuidados após a alta hospitalar.

Relativo à caracterização dos enfermeiros, notou-se que a pós-graduação foi cursada nas diferentes áreas do conhecimento, sobretudo pediatria e gerenciamento, indo ao encontro do perfil e das necessidades da clientela atendida na área de abrangência do serviço.

Com base nos achados da dimensão de estrutura, verificou-se que referente a recursos humanos, os usuáriosacompanhantes consideraram adequado o quantitativo de pessoal de enfermagem presente nas unidades pediátricas. Por outro lado, não houve consenso entre concordantes e discordantes nessa assertiva por parte dos enfermeiros. 
Nas organizações, a alocação de recursos humanos tem sido foco de atenção e motivo de instalação de conflito entre enfermeiros e administradores dos serviços de saúde, por interferir diretamente na qualidade e no custo. Assim, torna-se imperativo que o enfermeiro explicite as reais condições de assistência e empregue uma metodologia de dimensionamento de pessoal que possibilite a argumentação e a justificativa de suas respostas frente a um quadro de pessoal adequado, capaz de prestar uma assistência segura e humanizada ${ }^{(11)}$.

Com relação aos recursos materiais, verificou-se que não foi percebida, por parte da maioria dos participantes a escassez desses recursos.

O gerenciamento de recursos materiais envolve o processo de aquisição e disponibilização de materiais manufaturados, essenciais para a produção dos serviços de saúde. Dessa forma, o enfermeiro tem o papel preponderante na determinação do material necessário à consecução da assistência, seja na dimensão quantitativa, definindo as especificações técnicas e participando da compra, do controle e da avaliação desses insumos ${ }^{(12)}$.

Quanto ao mobiliário, observou-se que os acompanhantes-usuários também não demonstraram descontentamento. Todavia, os enfermeiros apontaram para a necessidade de um maior investimento nesse item.

No que tange à dimensão de processo, os achados pertinentes à interação de acompanhantes-usuários com a equipe de enfermagem mostrou ser da maior relevância, tendo em vista o grau de concordância nas respostas. Compartilhar saberes e espaços, não é um ato linear, decorrente da adesão a um discurso, e sim, implica mudanças de atitudes por parte dos pais e profissionais ${ }^{(13)}$.

O processo comunicativo é influenciado pelo clima interno e externo, apontando que, no interno, incluem-se os valores, os comportamentos e o nível de estresse do emissor e do receptor. Assim, no externo, nas variáveis envolvidas encontram-se o ambiente, o status, o poder e a autoridade ${ }^{(14)}$. Neste estudo, observou-se que a comunicação vem atendendo às demandas existentes na interface entre usuários e profissionais de saúde.

Em sua maioria, os respondentes consideraram que as orientações de alta atendiam às necessidades dos usuários. Pesquisas demonstram ser o enfermeiro o profissional da saúde melhor capacitado para elaborar um plano de alta, uma vez que permanece continuamente com o usuário e sua família, durante todo o período de internação ${ }^{(15)}$.

Nesta investigação, os registros de enfermagem mostraram-se satisfatórios, tanto para os enfermeiros como aos acompanhantes usuários.

Tendo em vista que, por meio dos registros de enfermagem, é possível mensurar indiretamente a qualidade assistencial e que esses se constituem em documentos técnicos e legais, torna-se fundamental conscientizar e empreender esforços, no sentido de que a equipe de enfermagem registre as atividades de maneira completa, a fim de traduzir o cuidado prestado ${ }^{(16)}$.

Os achados referentes à dimensão de resultado dizem respeito à satisfação com a assistência prestada, a importância da participação do usuário no tratamento, bem como a necessidade de orientação para a alta. Cabe destacar que conhecer e valorizar a opinião do acompanhante-usuário guarda estreita relação com a avaliação da qualidade dos serviços, sobretudo quando essas geram ações efetivas de melhoria da qualidade.

A satisfação em relação à assistência apresentou elevado grau de concordância parcial entre os enfermeiros, denotando que esses apresentam certas restrições nessa proposição.

A polissemia da qualidade impõe reconhecer os processos simbólicos e as práticas discursivas dos atores - sobretudo os usuários, impondo-se a valorização de suas percepções, entendendo-as não como subjetividades descontextualizadas, mas, como sinalização de experiências materializadas nas relações estabelecidas com as práticas de saúde ${ }^{(17)}$.

O conceito de satisfação é pluridimensional, pois engloba inúmeros fatores como as experiências adquiridas e os valores, tanto para o indivíduo como à sociedade.

Quanto à satisfação do usuário, esta é caracterizada como um indicador de resultados, associando-se à efetividade do cuidado ou a um ganho em uma dada intervenção ${ }^{(18)}$.

Em relação à orientação para a alta o maior percentual das respostas dos acompanhantes-usuários esteve no grau de indiferente, necessitando de melhor investigação.

Frente aos achados e como as três dimensões, embora sejam interdependentes, porém encontrem-se intimamente relacionadas, torna-se imperativo o monitoramento das mesmas, a fim de evitar repercussões indesejáveis à qualidade assistencial.

Decorrente das especificidades do processo de produção em saúde, nem uma estrutura adequada garante bons resultados e nem uma estrutura ruim impede; contudo, processos desenvolvidos com rigor da técnica podem atingir os resultados com excelência. Assim, as atuações estão sempre nos limites das possibilidades, pois uma estrutura adequada favorece processos adequados, que por sua vez, aumentam a probabilidade de resultados desejáveis $^{(19)}$.

\section{CONCLUSÕES}

Esta investigação possibilitou analisar a qualidade da assistência prestada em Unidades Pediátricas de um hospital de ensino, frente ao modelo donabediano, que contempla as dimensões de estrutura, processo e resultado.

Os quesitos que apresentaram diferença estatística 
significante na dimensão de estrutura, foram os referentes a recursos humanos (quantitativo de pessoal), materiais (insumos existentes) e hotelaria (mobiliário); Em relação à dimensão de processo, o relacionamento dos usuários com a equipe e a relevância dos registros de enfermagem e quanto à dimensão de resultado, foram observadas questões relativas à importância das orientações para alta e satisfação com a assistência.

No que tange à favorabilidade dos escores das três

\section{REFERÊNCIAS}

1. Donabedian A. Evalución de la calidad de la atención médica. In: White KL, Frank J, organizadores. Investigaciones sobre serviços de salud: uma antologia. Washington (DC): OPAS; 1992. p. 382-404.

2. Ramírez Sánches TJ, Nájera Aguila P, Nigenda Lopez G. Percepción de la calidad de la atención de los servicios de salud en México: perspectiva de los usuarios. Salud Pública Méx. 1998;40(1):3-12.

3. Patton MQ. Practical evaluation. Beverly Hill: Sage Publications; 1982.

4. Gouveia GC, Souza WV, Luna CF, Souza-Júnior PRB, Szwarcwald CL. Satisfação dos usuários do sistema de saúde brasileiro: fatores associados e regionais. Rev Bras Epidemiol. 2009;12(3):281-96.

5. Tanaka OY, Melo C. Avaliação de programas de saúde do adolescente: um modo de fazer. São Paulo: EDUSP; 2001.

6. Donabedian A. The role of outcomes in quality assessment and assurance. QRB Qual Rev Bull. 1992;18(11):356-60. Comment in: QRB Qual Rev Bull. 1993;19(3):78.

7. Adami NP, Yoshitome AY. Métodos de avaliação da assistência de enfermagem. Rev Bras Enferm. 2003;56(1):52-6.

8. Pereira JCR. Análise de dados qualitativos: estratégias metodológicas para as ciências da saúde, humanas e sociais. 3a. ed. São Paulo: EDUSP; 2001.

9. Sabatés AL, Borba RIH. As informações recebidas pelos pais durante a hospitalização do filho. Rev Latinoam Enferm. 2005;13(6):968-73.

10. Alves CA, Deslandes SF, Mitre RMA. Desafios da humanização no contexto do cuidado da enfermagem pediátrica de média e alta complexidade. Interface Comum Saúde Educ. 2009;13(Supl 1):581-94.

11. Gaidzinski RR, Fugulin FMT, Castilho V. dimensões avaliadas, verificou-se que a de estrutura obteve o menor escore, para os usuários e a de processo, para os enfermeiros. Diante desses resultados, infere-se que os usuários tendem a avaliar aspectos tangíveis e os profissionais, o próprio processo de trabalho.

Por conseguinte, este estudo propiciou ainda a identificação de problemas, permitindo aos gestores da instituição em questão a implementação de ações que contribuam para a melhoria contínua da qualidade.

Dimensionamento de pessoal de enfermagem em instituições de saúde. In: Kurcgant P, coordenadora. Gerenciamento em enfermagem. Rio de Janeiro: Guanabara Koogan; 2005. p. 125-37.

12. Castilho V, Gonçalves VM. Gerenciamento de recursos materiais. In: Kurcgant P, coordenadora. Gerenciamento em enfermagem. Rio de Janeiro: Guanabara Koogan; 2005. p.157-70.

13. Lima RAG, Rocha SMM, Scochi CGS. Assistência à criança hospitalizada: reflexões acerca da participação dos pais. Rev Latinoam Enferm. 1999;7(2):33-9.

14. Marquis BL, Huston CJ. Administração e liderança em enfermagem: teoria e prática. 4a. ed. Porto Alegre: Artmed; 2005.

15. Policastro S. Orientação de enfermagem: uma estratégia para minimizar a ansiedade e eventuais intercorrências imediatas à alta hospitalar de pacientes mastectomizadas [dissertação]. São Paulo: Escola de Enfermagem da Universidade de São Paulo; 2002.

16. Labbadia LL, Adami NP. Avaliação das anotações de enfermagem em prontuários de um hospital universitário. Acta Paul Enferm. 2004;17(1):55-62.

17. Bosi MLM, Uchimura KY. Avaliação da qualidade ou avaliação qualitativa do cuidado em saúde? Rev Saúde Pública $=\mathrm{J}$ Public Health. 2007;41(1):150-3.

18. Donabedian A. La calidade de la atención médica: definición y métodos de evaluación. México: Prensa Medica Mexicana; 1984.

19. Malik AM. Qualidade e avaliação nos serviços de saúde: uma introdução. In: D’Innocenzo M, coordenadora. Indicadores, auditorias, certificações: ferramentas de qualidade para gestão em saúde. São Paulo: Martinari; 2006. p. 21-35. 